\title{
GuideToPharmacology.org - an update
}

\section{Correspondence}

Stephen PH Alexander, School of Biomedical Sciences, University of Nottingham Medical School, Nottingham NG7 2UH, UK. E-mail: Steve.alexander@nottingham.ac.uk

\author{
Stephen PH Alexander ${ }^{1}$, Helen E Benson ${ }^{2}$, Anthony Davenport ${ }^{3}$, \\ Alistair Mathie ${ }^{4}$, Ian JC McGrath ${ }^{5}$, Adam J Pawson ${ }^{2}$, John A Peters ${ }^{6}$, \\ Joanna L Sharman ${ }^{2}$, Michael Spedding ${ }^{7}$ and Anthony J Harmar ${ }^{2}$ \\ ${ }^{1}$ School of Biomedical Sciences, University of Nottingham Medical School, Nottingham, UK, ${ }^{2}$ The \\ University/BHF Centre for Cardiovascular Science, University of Edinburgh, Edinburgh, UK, ${ }^{3}$ Clinical \\ Pharmacology Unit, University of Cambridge, Addenbrooke's Centre for Clinical Investigation, \\ Cambridge, UK, ${ }^{4}$ Medway School of Pharmacy, Universities of Kent and Greenwich, Medway, UK, \\ ${ }^{5}$ School of Life Sciences, University of Glasgow, Glasgow, UK, ${ }^{6}$ Neuroscience Division, Medical \\ Education Institute, Ninewells Hospital and Medical School, University of Dundee, Dundee, UK, and \\ ${ }^{7}$ Laboratoires Servier, Suresnes, France
}

\section{The website}

The website http://www.GuideToPharmacology.org is an online database of key pharmacological targets. The listings of 363 GPCRs, 91 transmitter-gated channels, 122 ion channels and 48 nuclear receptors were seeded from, and published online simultaneously, with the November 2011 edition of the Guide to Receptors and Channels (Alexander et al., 2011). The website, jointly funded initially by the British Pharmacological Society (BPS; http://www.bps.ac.uk) and the International Union of Basic and Clinical Pharmacology (http://www.iuphar.org), provides a readily searchable alternative to the paper-based version of the Guide to Receptors and Channels. Furthermore, the dovetailing with the IUPHAR database (http://www.iuphar-db.org, Sharman et al., 2011) provides a significant extension to the available resources. Thus, the primary aim of the Guide to Receptors and Channels is to provide a simple overview of pharmacological targets, where families are grouped together for ready comparison of individual members, as much as possible on a single page. The focus is deliberately on human proteins, identifying selective agents that allow definition of each pharmacological family and individual targets. A key feature is to pick out those agents that are available commercially or by donation, in order that, for example, a researcher new to the area of endothelin receptors can rapidly select compounds that permit characterization of individual subtypes, or equally important on occasion, to exclude the involvement of endothelin receptors altogether from an observed tissue response.
The IUPHAR database, on the other hand, provides a much greater level of detail. For example, individual records include multiple synonyms by which the target is known, together with links to multiple online databases listing structural information on human and non-human species, principally rat and mouse. Additionally, the IUPHAR database contains extended pharmacological information, where available, listing multiple ligands with additional information on assay conditions.

The backing of these two organizations ensures that the content is the authoritative voice on nomenclature and pharmacology. In addition, the involvement of the BPS and IUPHAR means that the information is available online without restriction, so that colleagues across the globe can have access to the most up-to-date information on these key pharmacological targets.

\section{The update}

The most recent updates on the GuideToPharmacology.org website allow oversight of 236 catalytic receptors (including cytokine receptors, natriuretic peptide receptors and receptor serine/threonine kinases), 500 solute transporters (including ATP-binding cassette transporters, P-type ATPases and solute carrier families) and 460 selected enzymes (including those involved in cyclic nucleotide, endocannabinoid and glycerophospholipid turnover).

One significant feature, which has not previously been possible with hard copies of the Guide to Receptors and 
Channels, is the complete ligand list. These are divided into lists of synthetic organics, metabolites, natural products, endogenous peptides, other peptides, inorganics, antibodies, and others. Individual pages show two-dimensional structures and simple physical-chemical properties. Where possible, biological activity is listed for the chemicals as substrates for enzymes or transporters, or modulators of receptors, channels, etc.

\section{The future}

The recent award of a Biomedical Resource Grant by the Wellcome Trust to Tony Harmar (PI), Stephen Alexander, Anthony Davenport and John Peters, together with continued support from IUPHAR and BPS, allows the project team (the Editors of the Guide to Receptors and Channels, members of the Nomenclature Committee of IUPHAR and the curators based at the University of Edinburgh, see Figure 1) to set ambitious goals for the future. Over the next 3-4 years the resource will be expanded to provide: (i) quantitative pharmacological information on all of the (human) targets of current prescription medicines and other likely targets of future small molecule drugs (at present, the targets of about half of all current licensed drugs are included); (ii) enhanced information on the therapeutic uses of drugs in the resource; and (iii) rigorous curation of the structure and nomenclature of the chemical substances in the resource, in collaboration with other chemistry databases (DrugBank, the Human Metabolome Database, ChemSpider, ChEMBL and PubChem).

You can keep up to date with additions to the databases by following the Twitter links @IUPHARDB, $@$ GuidetoPHARM, and, of course, @BritPharmSoc.

Web user

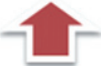

Web interface
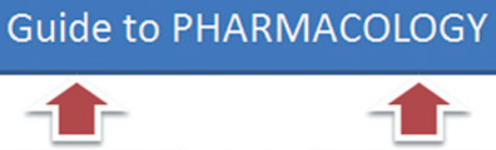

Databases

IUPHAR-DB

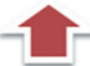

NC-IUPHAR

Data collection

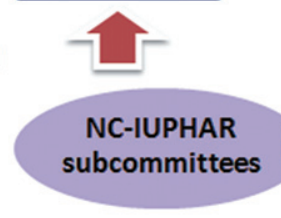

Figure 1

The organization of the website.

\section{References}

Alexander SPH, Mathie A, Peters JA (2011). Guide to receptors and channels (GRAC), 5th edn. Br J Pharmacol 164 (Suppl 1): S1-S324.

Sharman JL, Mpamhanga CP, Spedding M, Germain P, Staels B, Dacquet C et al. (2011). IUPHAR-DB: new receptors and tools for easy searching and visualization of pharmacological data. Nucleic Acids Res 39: D534-D538. 